\title{
Telomere elongation in parthenogenetic stem cells
}

\section{Dear Editor,}

Parthenogenetic embryonic stem ( $\mathrm{pES}$ ) cells, generated from oocytes by artificial activation without involvement of fertilization, show differentiation and pluripotency as evidenced by their capacity to generate germline chimeras and all pES pups by tetraploid embryo complementation, indicating the ability of pES cells to form all cell types in the body (Chen et al., 2009; Liu et al., 2011). Indeed, pES cells can repair injured muscle (Koh et al., 2009) and cardiomyocytes with reduced risk of tumorigenesis (Liu et al., 2013), and contribute to long-term hematopoiesis (Eckardt et al., 2007), supporting the potential applications of pES cells in cell transplantation therapy and tissue engineering (Koh et al., 2009). Furthermore, successful derivation of human pES cells provides important pluripotent stem cell sources alternative to ES cells (or fES, ES cells derived from fertilized embryos) for future clinic therapeutic uses (Mai et al., 2007). Telomere length maintenance is critical for genomic stability, unlimited self-renewal, and developmental pluripotency of ES cells. It remains elusive whether telomeres are sufficiently reprogrammed in pES cells.

We thought to analyze the telomere lengths of $p E S$ cells, characteristic of ES cells in morphology (Fig. 1A), in comparison with those of ES cells at similar passages. ES and pES cells were depleted off mouse embryonic fibroblasts (MEF) as feeder prior to harvest for analysis in subsequent experiments. We show that telomeres elongated, and were even slightly longer in pES than in ES cells. Two different pES cell lines (C3 and 1116) exhibited longer telomeres than did ES cells with identical genetic background estimated initially by telomere qPCR analysis (Fig. 1B), and also by quantitative telomere FISH (QFISH) method (Fig. 1C and 1D). Moreover, telomeres of pES cells elongated slightly during passages, like those of ES cells (BF10). The telomere QFISH data were generally consistent with relative telomere length expressed as T/S ratio by $\mathrm{qPCR}$. Also, two other $\mathrm{pES}$ cell lines generated from oocytes of inbred young C57BL/6 mice displayed telomere maintenance or elongation during passages, like fES cells (N33) (Fig. 1E). Together, telomeres are reprogrammed and sufficiently elongated in $\mathrm{pES}$ cells.

To investigate the molecular bases of differential telomere elongation, we performed global gene expression analysis of pES cells, compared with fES cells by microarray. Genes important for development and differentiation showed no or only minimal differences in their expression between $\mathrm{pES}$ and fES cells, and were not enriched in the differentially expressed gene lists (Tables S1 and S2). Expression of genes associated with pluripotency of ES cells, such as Pou5f1 (Oct4), Nanog, Sox2, and Rex1 did not differ among these three cell lines. Major telomerase genes Tert and Terc also did not show differential expression between pES and ES cells.

Interestingly, most of the up-regulated genes in both $\mathrm{pES}$ cell lines were enriched in 2-cell embryo state, including Tcstv1/3, Dub1, Eif1a, Gm4340, and Zscan4 (Zalzman et al., 2010; Macfarlan et al., 2012). Differential gene expression profile also was found between two pES cell lines, but pES cells 1116 closely resembled ES cells more than did pES cells C3 (Fig. 1F). For instance, Lefty1 and Stella (also known as Dppa3), expressed at reduced levels in pES cells $\mathrm{C} 3$, compared with pES 1116 or ES cells. Coincidently, chimeras generated from pES 1116 but not C3 showed germline competency (Liu et al., 2011). The microarray data were validated by qPCR analysis of selected genes, although the fold in relative expression levels showed some differences for a few genes (Fig. 1G).

By immunofluorescence microscopy, Zscan4 was expressed sporadically in only small proportion $(1 \%-5 \%)$ of ES cell cultures, consistent with the reports (Zalzman et al., 2010; Macfarlan et al., 2012). While some of Zscan4 positive ES cells were excluded from Oct4 expression, a few others showed weak positive staining for Oct4 (Fig. 2A). In addition, the proportion of Zscan4 positive cells was increased in pES cells, with higher ratio in pES C3 by both flow cytometry and immunofluorescence microscopy quantification (Fig. 2A-C). The protein levels of Zscan4 also were higher in pES cells than in ES cells, and highest in pES cell C3 (Fig. 2D), consistent with flow cytometry and immunofluorescence quantification data. Moreover, murine endogenous virus element (MERV) expressed at higher levels in pES than in ES cells by qPCR (Fig. S1A and S1B).

Zscan4 was shown to lengthen telomeres of ES cells presumably via recombination based mechanism (Zalzman et al., 2010). To examine whether elevated levels of Zscan4 also are implicated in telomere elongation of pES cells, we knocked down Zscan4 in two pES cell lines by two independent shRNAs effectively targeting Zscan4 (Fig. 2E). Depletion of Zscan4 shortened telomeres in both pES cell 
A

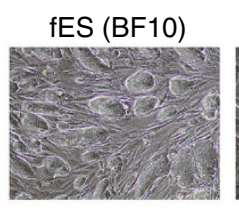

C

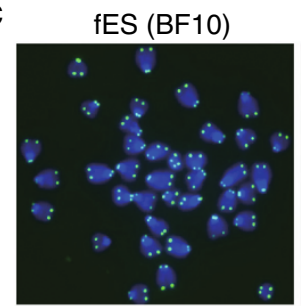

D
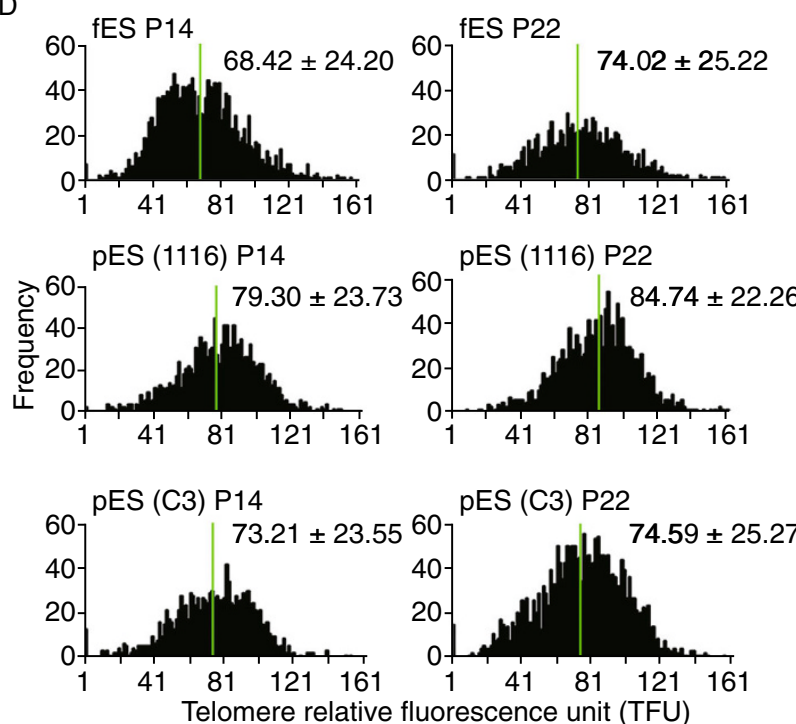

pES (C3) P22

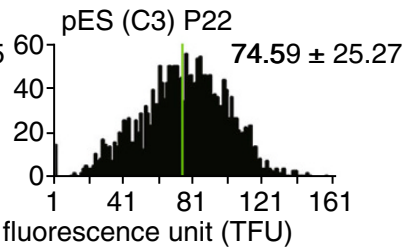

$\mathrm{G}$

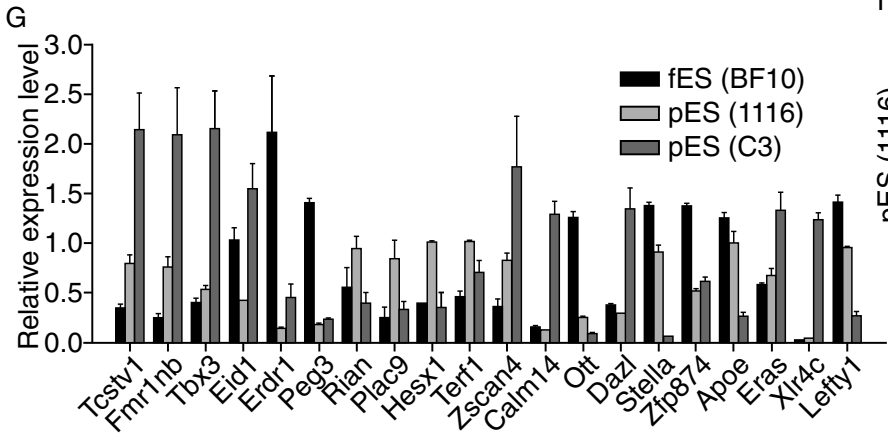

B
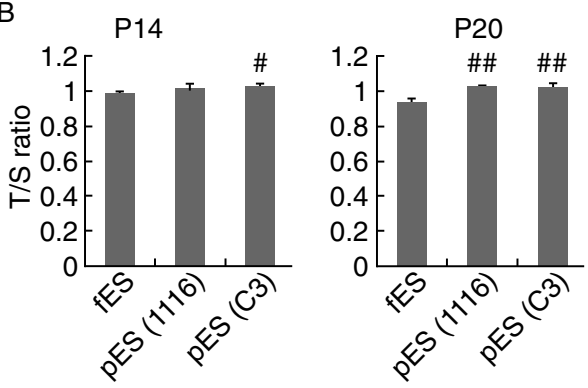

E

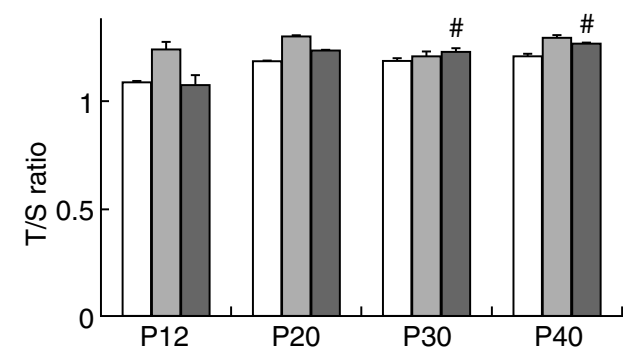

$\mathrm{F}$
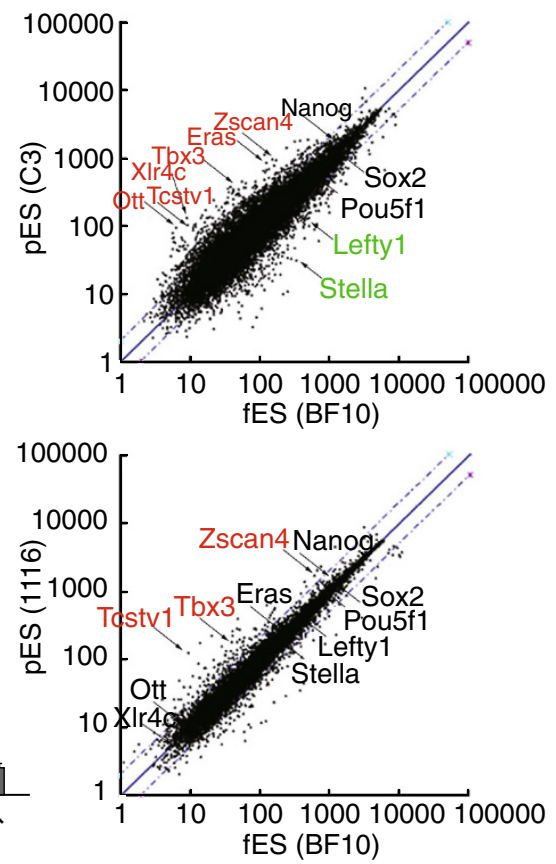

Figure 1. Telomere length and genome-wide gene expression of pES cells versus ES (fES) cells. (A) Colony morphology of pES cells $(1116, C 3)$ and fES cells (BF10) at passages 13-15. (B) Relative telomere length expressed as T/S ratio measured by quantitative real-time PCR method. Error bars indicate mean $\pm \mathrm{SD}$ (at least two repeats). ${ }^{*}, P<0.05 ;{ }^{* *}, P<0.01$, compared to fES cells. (C) Telomere quantitative FISH images of chromosome spread from pES and fES cells. Green dots, telomeres; blue, DAPIstained chromosomes. (D) Distribution histogram showing relative telomere length (TFU) of pES cells and fES cells, analyzed by telomere Q-FISH and TFL-TELO software (10-15 spreads analyzed for each cell line). (E) Longer telomere length expressed as T/S ratio estimated by qPCR in $\mathrm{pES}$ (Y5 and $\mathrm{Y} 6$ ) derived from oocytes of C57BL/6 mice, compared with fES (N33) from the same genetic background. * $P<0.05$, compared to the corresponding pES at P12. (F) Scatter plot showing comparison of global gene expression of $\mathrm{pES}$ cells and fES cells. Genes up-regulated (highlighted in red) and down-regulated (in green) in pES cells (C3 and 1116) were compared with those of fES cells. Genes are listed in Tables S1 and S2 using cut off as fold $\geq 2.0$. (G) Real-time PCR validation of selected 20 genes differentially expressed between $\mathrm{pES}$ and fES cells by microarray. 
A

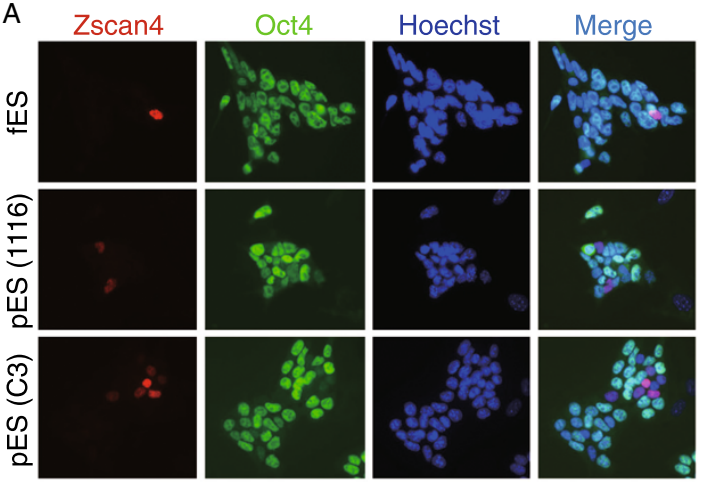

E

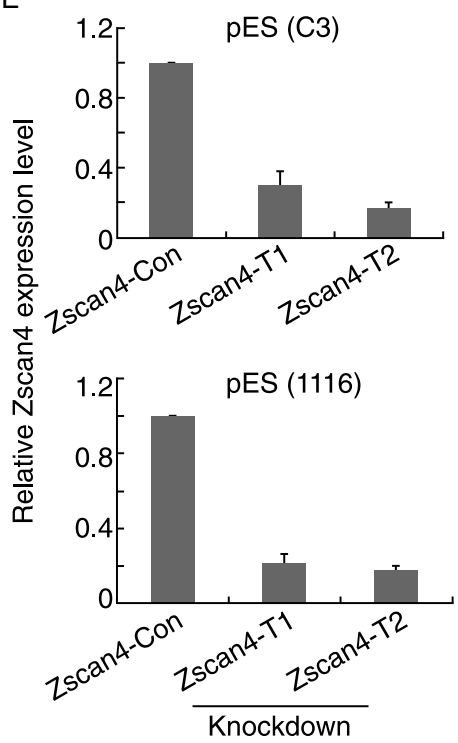

B
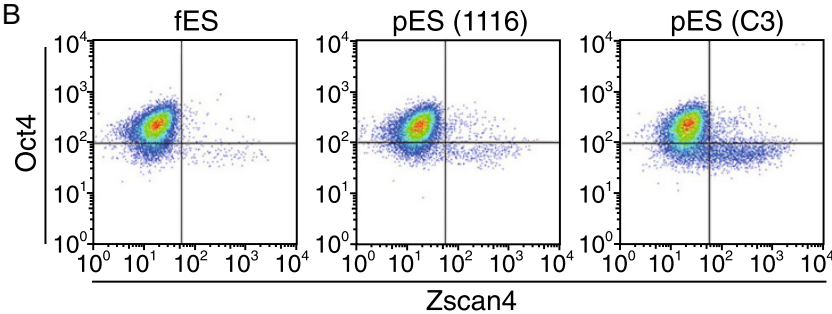

$\mathrm{C}$

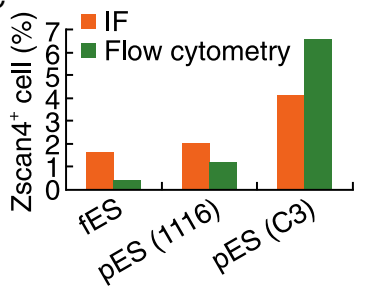

$\mathrm{D}$

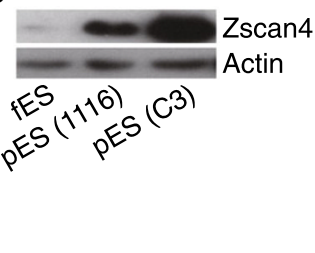

F

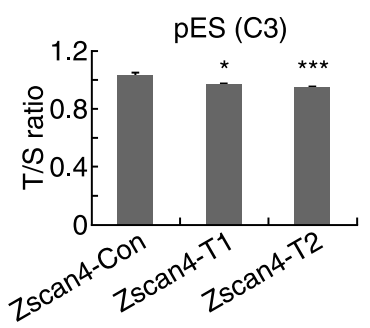

pES (1116)

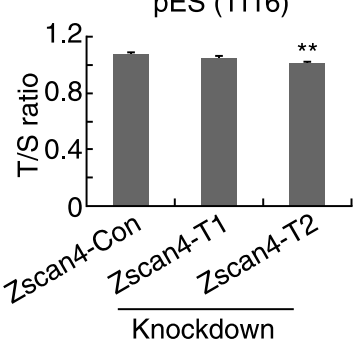

G
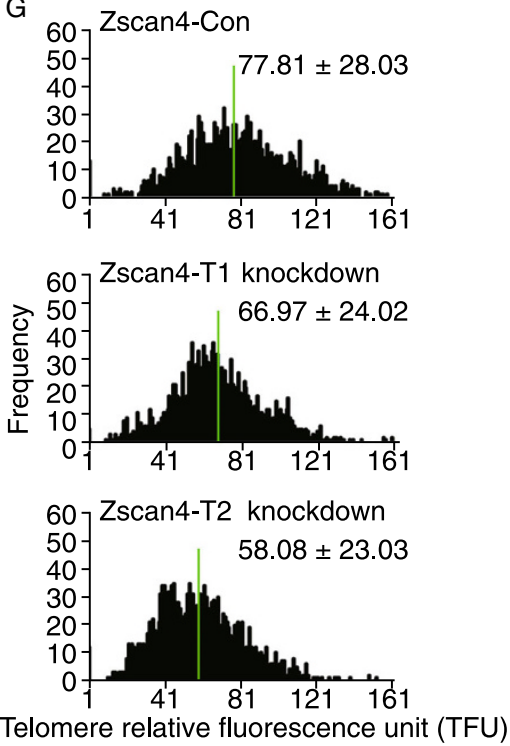

Figure 2. Involvement of Zscan4 in telomere elongation of pES cells. (A) Immunofluorescence images of Zscan4 (red) and Oct4 (green) in pES (1116 and C3) versus fES cells (BF10) at passage 15-16. Nuclei stained with Hoechst 33342 (blue). (B) Analysis of Zscan $4^{+}$cells in pES or fES cell cultures at passage $15-16$ by flow cytometry. Two repeats. (C) Proportion of Zscan $4^{+}$cells in pES cell cultures (1116 and C3) compared with fES cell cultures (BF10) by flow cytometry and immunofluorescence microscopy. (D) Protein levels of Zscan4 in fES cells (BF10) and pES cells (1116 and C3) at passage 15-16 by Western blot. $\beta$-Actin served as loading control. (E) Zscan4 expression levels by qPCR are notably lower in Zscan4 stable knockdown (KD) pES cells (C3 or 1116) by two shRNAs (T1, T2), compared with knockdown controls. (F) Telomeres of Zscan4 knockdown (Zscan4-T1 and Zscan4-T2) pES cells (C3 or 1116) are shorter than those of control knockdown pES cells (Zscan4-Con) at passage 20 analyzed by qPCR. Error bars indicate mean $\pm \mathrm{SD} .{ }^{*}, P<0.05 ;{ }^{* *}, P<0.01 ;{ }^{* * *}, P<0.001$, compared with control knockdown. (G) Distribution histogram showing relative telomere length as TFU in Zscan4 knockdown (Zscan4-T1 and Zscan4-T2) pES cells (C3) and in control knockdown cells (Zscan4-Con) at passage 20, analyzed by telomere Q-FISH and the TFL-TELO software. Medium telomere lengths are indicated by green bar.

lines estimated by QFISH as well as qPCR (Fig. 2F and 2G), suggesting that Zscan 4 might involve in telomere elongation of $\mathrm{pES}$ cells. However, various telomere lengths of $\mathrm{pES}$ cells (Fig. 1B-D), did not completely correlate with absolute Zscan4 protein levels (Fig. 2D), suggesting that genes other than Zscan 4 may also play roles in telomere elongation and self-renewal of $\mathrm{pES}$ cells.
Moreover, epigenetic modifications at telomeres and subtelomeres regulate telomere lengths (Blasco, 2007). Active histones $\mathrm{H} 3 \mathrm{~K} 4 \mathrm{me} 3, \mathrm{H} 3 \mathrm{~K} 9 \mathrm{Ac}, \mathrm{H} 3 \mathrm{Ac}$ and repressive histone H3K27me3 mostly enriched in euchromatin did not show noticeable differences in their protein levels between $\mathrm{pES}$ and ES cells, whereas heterochromatic repressive $\mathrm{H} 3 \mathrm{~K} 9 \mathrm{me} 3$ levels were reduced in $\mathrm{pES}$ cells compared with 
ES cells (Fig. S1C). Lower levels of H3K9me3 may derepress Zscan4 and Tcstv1 located at subtelomeres.

Reduced MAPK and increased Wnt Signaling are implicated in derivation, self-renewal, and pluripotent state of mouse ES cells. Parthenogenetic 'blastocysts' display reduced MAPK and increased Wnt signaling (Liu et al., 2010) and this may contribute to more efficient derivation of $p E S$ cells in mice (Chen et al., 2009) as well as in human (Mai et al., 2007). Membrane $\beta$-catenin was found in both pES and ES cells (Fig. S2A), and nuclear $\beta$-catenin protein expressed at relatively higher levels in pES than in ES cells (Fig. S2B), although total $\beta$-catenin levels seemed not to differ between the two cell lines (Fig. S2C). Erk levels appeared slightly lower in pES 1116, relative to ES cells, but much lower in pES C3 (Fig. S2C), which showed highest Zscan4 levels.

Factors in oocytes or early cleavage embryos presumably contribute to both rapid telomere reprogramming and epigenetic reprogramming during early embryo development. These critical factors can be exploited to improve reprogramming induction of iPS cells. Notably, telomeres elongate slowly during iPS cell induction and gradually acquire ES cell telomere lengths during continued passages (Wang et al., 2012), different from rapid telomere lengthening in early cleavage embryos (Liu et al., 2007). Our data suggest that increased expression of 2-cell genes particularly Zscan4 may partially explain telomere elongation of pES cells. Also, Zscan4 proves to rapidly lengthen telomeres and greatly enhance genomic stability and quality of iPS cells (Jiang et al., 2013). Multiple factors can influence pluripotency and germline competency of ES/iPS/pES cells. The functional significance of telomere elongation in $\mathrm{pES}$ cells or slightly longer telomeres in $\mathrm{pES}$ cells relative to ES cells remains unclear, but speculatively the robust telomere maintenance may help maintenance of self-renewal of pES cells in vitro.

In summary, pES cells generated from parthenogenetically activated oocytes exhibit telomere elongation or even slightly longer telomeres compared with fES cells. Without complication of sperm factors, parthenogenetically activated oocytes themselves can effectively elongate telomeres during early cleavage development (Liu et al., 2007). Consistently, somatic cell nuclear transfer (SCNT) using oocytes further improves chromatin remodeling and telomere elongation of iPS cells (Liu et al., 2012). SCNT efficiency in cloning human embryos has been remarkably improved recently with success at long last, such that human SCNT ES cells now are effectively achieved (Tachibana et al., 2013). It would be interesting to further explore whether telomeres are effectively elongated to maintain genomic stability of SCNT ES cells achieved using oocyte factors.

Electronic supplementary material The online version of this article (doi:10.1007/s13238-013-0006-z) contains supplementary material, which is available to authorized users.

$\mathrm{Yu}$ Yin and $\mathrm{Na}$ Liu contributed equally to the work.

\section{FOOTNOTES}

We thank Maja Okuka for helping with telomere QFISH experiments and David Keefe for valuable discussion and input of the work, Siyuan Xia and Xinpeng Yao for helping with flow cytomety analysis. This work was supported by the National Basic Research Program (973 Program) (Nos. 2012CB911202, 2011CBA01002), and the National Natural Science Foundation of China (Grant No. 31271587).

Yu Yin, Na Liu, Xiaoying Ye, Renpeng Guo, Jie Hao, Fang Wang, and Lin Liu declare that they have no conflict of interest. This article does not contain any studies with human or animal subjects performed by any of the authors.

Yu Yin, Na Liu, Xiaoying Ye, Renpeng Guo, Jie Hao, Fang Wang, Lin Liu

State Key Laboratory of Medicinal Chemical Biology, Department of Cell Biology and Genetics, College of Life Sciences, Nankai

University, Tianjin 300071, China

$\bowtie$ Correspondence: liulin@nankai.edu.cn (L. Liu)

\section{OPEN ACCESS}

This article is distributed under the terms of the Creative Commons Attribution License which permits any use, distribution, and reproduction in any medium, provided the original author(s) and the source are credited.

\section{REFERENCES}

Blasco MA (2007) Nat Rev Genet 8:299-309

Chen Z, Liu Z, Huang J, Amano T, Li C et al (2009) Stem Cells 27:2136-2145

Eckardt S, Leu NA, Bradley HL, Kato H, Bunting KD et al (2007) Genes Dev 21:409-419

Jiang J, Lv W, Ye X, Wang L, Zhang M et al (2013) Cell Res 23:92106

Koh CJ, Delo DM, Lee JW, Siddiqui MM, Lanza RP et al (2009) Methods 47:90-97

Liu L, Bailey SM, Okuka M, Munoz P, Li C et al (2007) Nat Cell Biol 9:1436-1441

Liu N, Enkemann SA, Liang P, Hersmus R, Zanazzi C et al (2010) J Mol Cell Biol 2:333-344

Liu Z, Hu Z, Pan X, Li M, Togun TA et al (2011) Hum Mol Genet 20:1339-1352

Liu Z, Wan H, Wang E, Zhao X, Ding C et al (2012) Stem Cells Dev 21:3001-3009

Liu Y, Ye X, Mao L, Cheng Z, Yao X et al (2013) Cardiovasc Res 97:208-218

Macfarlan TS, Gifford WD, Driscoll S, Lettieri K, Rowe HM et al (2012) Nature 487:57-63

Mai Q, Yu Y, Li T, Wang L, Chen MJ et al (2007) Cell Res 17:10081019

Tachibana M, Amato P, Sparman M, Gutierrez NM, Tippner-Hedges $R$ et al (2013) Cell 153:1228-1238

Wang F, Yin Y, Ye X, Liu K, Zhu H et al (2012) Cell Res 22:757-768

Zalzman M, Falco G, Sharova LV, Nishiyama A, Thomas $M$ et al (2010) Nature 464:858-863 